Lipid topogenesis - 35 years on

\title{
Lipid topogenesis - 35 years on
}

Neha Chauhan ${ }^{1 \#, ~ L u c e ~ F a r i n e ~}{ }^{2 \#}$, Kalpana Pandey ${ }^{1 \#, ~ A n a n t ~ K . ~ M e n o n}{ }^{1 \uparrow}$ and Peter Bütikofer ${ }^{2 \uparrow}$

${ }^{1}$ Department of Biochemistry, Weill Cornell Medical College, New York, 10065, USA

${ }^{2}$ Institute of Biochemistry and Molecular Medicine, University of Bern, Bern, Switzerland

\# Equal contributions (listed alphabetically)

ף Corresponding authors

Correspondence:

Peter Bütikofer, Ph.D., Institute of Biochemistry and Molecular Medicine, University of Bern, Bühlstrasse 28, 3012 Bern, Switzerland

Phone: +41316314113 / E-mail: peter.buetikofer@ibmm.unibe.ch

Anant K. Menon, Ph.D., Department of Biochemistry, Weill Cornell Medical College, 1300 York Avenue, NY 10065, New York, USA

Phone: +1 2127465941 / E-mail: akm2003@med.cornell.edu

\section{Keywords}

CDP-alcohol, endoplasmic reticulum, Kennedy pathway, membrane protein, phospholipid, scramblase

(C) 2016. This manuscript version is made available under the Elsevier user license http://www.elsevier.com/open-access/userlicense/1.0/ 
Lipid topogenesis - 35 years on

\begin{abstract}
Abbreviations:
C/EPT, choline/ethanolamine phosphotransferase; CDP, cytidine diphosphate; CDS, CDPDAG synthase; CL, cardiolipin; DGAT, diacylglycerol acyltransferase; DIPPS, di-myoinositol-1,3'-phosphate-1'-phosphate synthase; GPCR, G protein-coupled receptor; GPI, glycosylphosphatidylinositol; IPCT, L-myo-inositol-1-phosphate cytidylyltransferase; PA, phosphatidic acid; PC, phosphatidylcholine; PE, phosphatidylethanolamine; PG, phosphatidylglycerol; PI, phosphatidylinositol; PIPS, phosphatidylinositol-phosphate synthase; PIS, phosphatidylinositol synthase.
\end{abstract}




\begin{abstract}
Glycerophospholipids are the principal fabric of cellular membranes. The pathways by which these lipids are synthesized were elucidated mainly through the work of Kennedy and colleagues in the late 1950s and early 1960s. Subsequently, attention turned to cell biological aspects of lipids: Where in the cell are lipids synthesized? How are lipids integrated into membranes to form a bilayer? How are they sorted and transported from their site of synthesis to other cellular destinations? These topics, collectively termed 'lipid topogenesis', were the subject of a review article in 1981 by Bell, Ballas and Coleman. We now assess what has been learned about early events of lipid topogenesis, i.e. "lipid synthesis, the integration of lipids into membranes, and lipid translocation across membranes", in the 35 years since the publication of this important review. We highlight the recent elucidation of the X-ray structures of key membrane enzymes of glycerophospholipid synthesis, progress on identifying lipid scramblase proteins needed to equilibrate lipids across membranes, and new complexities in the subcellular location and membrane topology of phosphatidylinositol synthesis revealed through a comparison of two unicellular model eukaryotes.
\end{abstract}




\section{Introduction}

Thirty-five years ago, Bell, Ballas and Coleman published a review article entitled 'Lipid topogenesis' [1]. The previous year, Blobel had published an article entitled 'Intracellular protein topogenesis' [2] (see also the related review by Rothman and Lenard [3]). Both articles focused on the endoplasmic reticulum (ER), a biogenic membrane capable of synthesizing and integrating its lipid and protein components. The term topogenesis is not found in the Oxford English Dictionary but was used in both papers to describe events that accompany and follow the synthesis of membrane components. Early topogenic events comprise those that occur concurrently with - or soon after - the synthesis of lipids and proteins, whereas late events refer to the processes whereby these molecules are sorted and exported from the ER to reach other cellular destinations. These are fundamental processes that describe the biogenesis of cellular membranes.

Cells synthesize a diverse array of glycerophospholipids and other lipids. The question of why there are so many lipid species has been discussed [4-6]. Thus, lipid diversity is necessary to generate thin and thick cell membranes with biogenic and barrier functions, provide transbilayer charge asymmetry, endow membranes with adaptive properties that allow them to tolerate changes in temperature by eliminating phase transitions, enable membranes to curve, bend and be sculpted into vesicles, provide a wide range of signaling molecules, and support the activities of membrane proteins many of which require a particular lipid environment and specific lipid cofactors. The diverse spectrum of cellular lipids is synthesized via complex, sometimes parallel and often essential pathways, and lipid diversity is increased and homeostatically maintained by remodeling reactions in which acyl chains are removed and replaced. The paradigmatic pathways of glycerophospholipid biosynthesis were elucidated in the late 1950s and early 1960s [7,8] and highlight the central role of cytidine nucleotides. These pathways, termed the Kennedy pathways, are shown in Figure 1. Biosynthesis requires CDP-alcohol phosphotransferase enzymes that catalyze the formation of a phosphodiester bond linking the head and tail components of the lipid. For this, the head or tail of the lipid enters the enzymatic reaction as an activated (high energy) precursor in the form of a CDP-alcohol. Transfer to a cognate acceptor alcohol (a tail or a head, respectively) results in the formation of a phospholipid. Thus, a water-soluble CDP-alcohol (headgroup donor) may be combined with 
diacylglycerol (DAG) as in the case of phosphatidylcholine (PC) synthesis. Alternatively, a lipophilic CDP-alcohol, CDP-DAG, may be used in conjunction with a non-activated headgroup as in the case of phosphatidylinositol (PI) synthesis. The reaction can be written generically as:

$$
\mathrm{CDP}-\mathrm{R}_{1}+\mathrm{R}_{2} \rightarrow \mathrm{R}_{1}-\mathrm{P}-\mathrm{R}_{2}+\mathrm{CMP}
$$

(where $\mathrm{R}_{1}$ and $\mathrm{R}_{2}$ correspond to the head and tail components of the phospholipid ( $\mathrm{R}_{1}$-P$\mathrm{R}_{2}$ ), or vice versa, and $\mathbf{P}$ indicates a phosphodiester bond).

The main conclusion of the Bell et al. review [1] was that CDP-alcohol phosphotransferase-catalyzed lipid biosynthetic reactions are carried out on the cytoplasmic face of the ER by membrane-bound enzymes. Thus lipid synthesis is asymmetric, resulting in the deposition of new phospholipids into the cytoplasmic leaflet of the ER membrane. There is no parallel biosynthetic pathway to generate phospholipids in the luminal leaflet of the ER. Thus, propagation of the lipid bilayer requires newly synthesized lipids to be translocated across the membrane to populate the luminal leaflet. As spontaneous transbilayer translocation of phospholipids does not occur at an appreciable rate, it was assumed that a lipid transporter - a scramblase protein - must be involved. Much of the experimental work supporting these conclusions was obtained via studies on sealed rat liver microsomes. Bell et al. discuss experiments where proteases and membrane impermeant inhibitors were used to probe the orientation of the active sites of lipid biosynthetic enzymes in microsomes, and in which the transport (or lack thereof) of substrates such as CDP-choline and palmitoyl-CoA into the vesicles was measured and localization of lipid products was determined by phospholipases or chemical modification. 


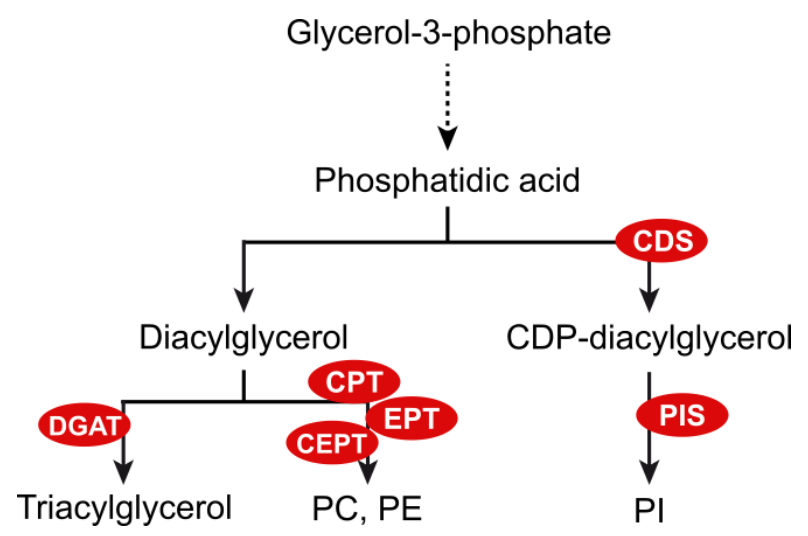

Figure 1. The Kennedy pathway of glycerophospholipid synthesis. Phosphatidic acid is synthesized in multiple steps from glycerol-3phosphate (obtained from glycolysis or the phosphorylation of glycerol) and fatty acyl-CoA. It is the precursor of diacylglycerol and the liponucleotide CDP-diacylglycerol, both of which are used for phospholipid biosynthesis.

Phospholipids are synthesized by pathways that use a CDP-alcohol as an activated precursor. The activated lipid tail CDP-diacylglycerol is used, for example, in the synthesis of phosphatidylinositol (PI) in eukaryotes and PI-phosphates in prokaryotes. Alternatively, a water-soluble CDP-alcohol, e.g. CDPcholine or CDP-ethanolamine, is combined with diacylglycerol to generate phosphatidylcholine (PC) or phosphatidylethanolamine (PE). Diacylglycerol is also used for the synthesis of the neutral lipid triacylglycerol. CDS, cytidine-diphosphate diacylglycerol synthase; CPT, cholinephosphotransferase; CEPT, choline/ethanolamine phosphotransferase; DGAT, diacylglycerol acyltransferase; EPT, ethanolamine phosphotransferase; PIS, phosphatidylinositol synthase.

Despite the concurrency of the two review articles on protein and lipid topogenesis, studies of protein topogenesis have yielded a wealth of information $[9,10]$ whereas progress on lipid aspects has been comparatively slow. However, with relatively recent advances in certain areas such as lipidomics $[5,11,12]$ and live cell imaging of lipids [13](Kobayashi, this special issue), the understanding of late lipid topogenic events has steadily improved. In contrast, early topogenic events remain poorly defined but the prospects for improvement here are bright. For example, in the past couple of years there has been an explosion of structural information on lipid biosynthetic enzymes [14-17] and while as yet there is no indication of the molecular identity of the scramblase that is needed to redistribute newly synthesized lipids between the two leaflets of the ER membrane [18], the recent discovery of other scramblase proteins [19-24] lends hope that the identity of the ER scramblase will soon be revealed. Here our objective is to review these recent advances and to assess what has been learned about early events of lipid topogenesis, i.e. 
'lipid synthesis, the integration of lipids into membranes, and lipid translocation across membranes' [1], in the 35 years since the publication of the review by Bell and colleagues.

\section{CDP-alcohol phosphatidyltransferase enzymes}

The Bell et al. review [1] is best known for capturing the concept that glycerophospholipids are synthesized on the cytoplasmic face of the ER, and that newly synthesized phospholipids must redistribute across the membrane in order to expand the bilayer uniformly. This conclusion is depicted pictorially in Figure 2, which also shows the best current assessment of the membrane topology of CEPT, the choline/ethanolamine phosphotransferase enzyme that generates phosphatidylcholine (PC) and phosphatidylethanolamine (PE) in the ER membrane from the corresponding CDP-alcohol and diacylglycerol (DAG). Active site residues of CEPT are found within a cytoplasmic loop between the first and second transmembrane domain. The choline-specific CPT enzyme is located in the Golgi apparatus, but has a similar predicted membrane topology [25]. PA provides DAG in the ER, whereas Golgi DAG may be a byproduct of sphingomyelin synthesis.

A

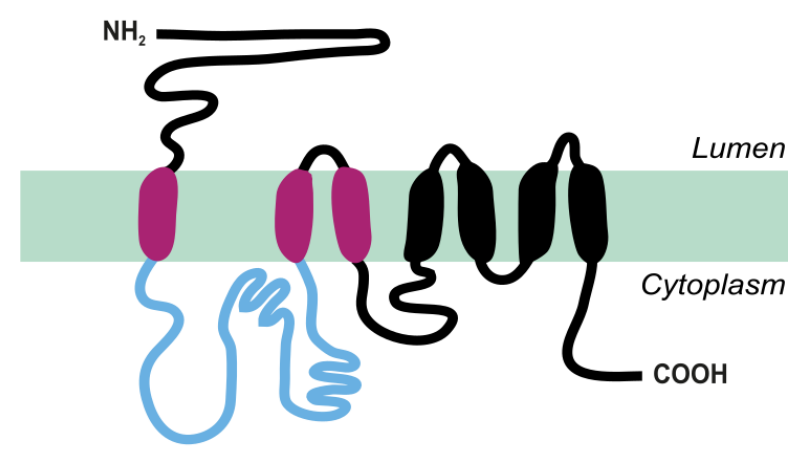

B

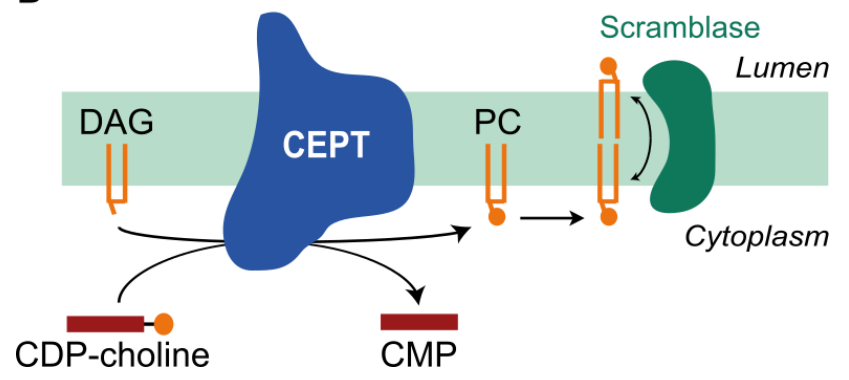

Figure 2. Asymmetric synthesis and transbilayer scrambling of phosphatidylcholine in the ER. A, Predicted membrane topology of human CEPT1 [25]. Regions of CEPT necessary for binding diacylglycerol (DAG) are shown in pink, and the amino acid stretch needed for CDP-alcohol binding and enzymatic activity is shown in light blue [25]. The light blue region also contains the consensus motif $D(x)_{2} D G(x)_{2} A R(x)_{7}$. ${ }_{12} G(x)_{3} D(x)_{3} D$ for this class of enzymes [15]. The ER membrane bilayer is shown as a green slab. $\boldsymbol{B}$, Phosphatidylcholine (PC) synthesis and ER bilayer assembly (adapted from [26]). CEPT uses DAG and CDP-choline to form PC on the 
cytoplasmic leaflet of the ER membrane. A scramblase is needed to equilibrate newly synthesized PC between the two leaflets of the membrane bilayer.

CEPT, CPT and EPT (Figure 1) are members of the CDP-alcohol phosphotransferase family. These enzymes catalyze the scission of a phosphoanhydride bond to release CMP from a CDP-alcohol, with the concomitant formation of a phosphodiester bond (Figure 2). Beyond scanning mutagenesis studies supplemented with bioinformatics to define active site residues, and the use of membrane topology prediction algorithms to determine the orientation of the active site with respect to the membrane (Figure 2)[25], no molecular information was available on this class of enzymes. However, the recent reports of the Xray structures of two CDP-alcohol phosphotransferases $[15,16]$ from the hyperthermophilic archaeon Archaeoglobus fulgidus that use a water-soluble CDP-alcohol (Figure 3) and another from the Gram-positive bacterium Renibacterium salmoninarum that uses CDP-DAG [17] shed light on the molecular basis of phospholipid biosynthesis.

In this section we focus on the $A$. fulgidus CDP-alcohol phosphotransferases AfAPT1 and AfAPT2, encoded by the genes AF0263 [15] and AF2299 [16], respectively. The $R$. salmoninarum enzyme will be discussed later. AfAPT1 is a bifunctional enzyme, organized in the form of two domains (Figure 3)[15,27]. The cytoplasmic domain (IPCT) synthesizes CDP-inositol, whereas the transmembrane domain (DIPPS) is a CDP-alcohol phosphotransferase. The specific reactions catalyzed by AfAPT1 are:

(i) Inositol-1-phosphate + CTP $\rightarrow$ CDP-inositol + PPi (catalyzed by L-myo-inositol-1phosphate cytidylyltransferase (IPCT))

(ii) CDP-inositol + inositol-1-phosphate $\rightarrow$ di-inositol-1,3'-phosphate -1'-phosphate + CMP (catalyzed by a di-myo-inositol-1,3'-phosphate-1'-phosphate synthase (DIPPS)).

As evident from the reaction sequence, AfAPT1 does not synthesize a phospholipid but rather DIPP, a water-soluble compound; DIPP is eventually dephosphorylated to the osmolyte di-inositol-1,3'-phosphate (DIP) which serves a protective role in hyperthermophiles. Although the substrate and product of DIPPS are water-soluble, the DIPPS domain of AfAPT1 is a polytopic membrane protein. It has been suggested that this is 
an evolutionary hold-over as DIPP-synthases evolved from phosphatidylinositol (PI) synthases [28] which are invariably membrane proteins.

AfAPT1 forms a dimer, with interactions between the two monomers occurring via hydrophobic contacts in the DIPPS domain within the lipid bilayer (Figure 3). The DIPPS domain comprises of six transmembrane $\alpha$-helices with polar and charged residues on the cytoplasmic side. These residues line a polar cavity that is accessible from the cytoplasm. The cavity harbors four aspartate residues that coordinate a $\mathrm{Mg}^{2+}$ ion required for catalysis. Three of the four aspartate residues belong to a previously described consensus motif $\left(\mathrm{DG}(\mathrm{x})_{2} \mathrm{AR}(\mathrm{x})_{8} \mathrm{G}(\mathrm{x})_{3} \mathrm{D}(\mathrm{x})_{3} \mathrm{D}\right)$ characteristic of all CDP-alcohol phosphotransferases and appear to directly coordinate the metal ion. Given its proximity to the other aspartates and $\mathrm{Mg}^{2+}$, and measured loss of enzymatic activity on replacement with alanine, the authors suggest that the fourth aspartate residue should also be included in this consensus motif, which they revised to the following form: $D(x)_{2} D G(x)_{2} A R(x)_{7-12} G(x)_{3} D(x)_{3} D$.

Two potential substrate-binding pockets were identified in the DIPPS domain using in silico methods (Figure 3). Pocket 1 is flanked by the CDP-alcohol phosphotransferase consensus motif. It is located on top of the central catalytic pocket close to the $\mathrm{Mg}^{2+}$ binding site and is proposed to be involved in binding CDP-alcohol. Pocket 2, located at the base of the hydrophilic cavity, is proposed to provide the binding site for inositol-1-phosphate based on sequence conservation. The proposed role of these binding pockets was confirmed by molecular docking experiments and mutagenesis studies [15]. Catalysis carried out by DIPPS occurs by a $\mathrm{S}_{\mathrm{N}} 2$ displacement mechanism where aspartate residues act as base catalysts to promote the nucleophilic attack of the - $\mathrm{OH}$ in inositol-1-phosphate on the $\beta$-phosphoryl of CDP-inositol to form DIPP and CMP.

A final point concerns the cytoplasmic IPCT domain (Figure 3). The architecture of this domain is characteristic of a nucleotidyltransferase Rossmann fold. It is interesting to note that the catalytic pocket of the IPCT domain faces away from the CDP-inositol binding pocket (pocket 1) of DIPPS even though the two domains have to catalyze consecutive steps of DIPP formation (Figure 3). It has been proposed that substrate binding brings about conformational changes that can orient the two domains such that their functions are coordinated. 
A

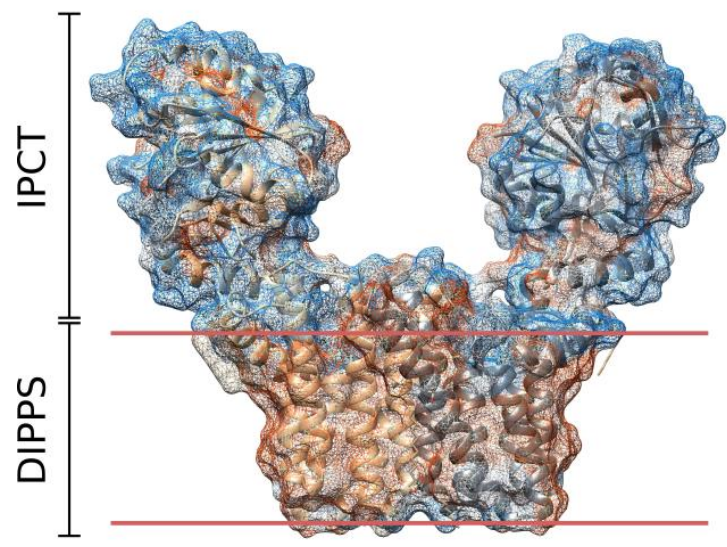

B

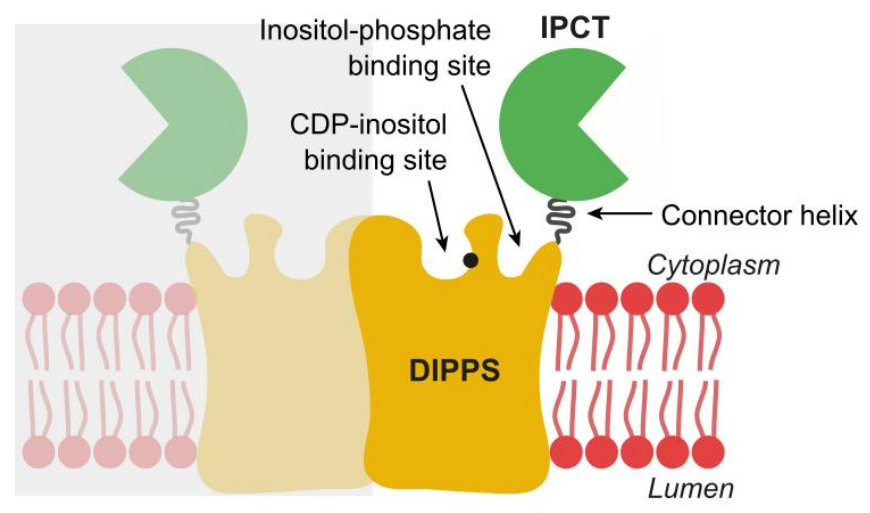

Figure 3. Structure of the AfAPT1 CDP-alcohol phosphotransferase. A, Three-dimensional structure of the AfAPT homodimer. Each monomer consists of a L-myo-inositol-1-phosphate cytidylyltransferase (IPCT) domain linked to a di-myo-inositol-1,3'-phosphate-1'-phosphate synthase (DIPPS) domain. The borders of the membrane bilayer are indicated as red lines. B, Cartoon representation of AfAPT1 showing the homodimeric protein in a membrane bilayer (phospholipids are in red). The IPCT domain in each monomer is linked via a connector helix to the transmembrane CDPalcohol phosphotransferase domain (DIPPS). Interactions between the DIPPS domains within the membrane mediate dimer formation. The binding pockets for CDP-inositol and Inositol-1-phosphate in the DIPPS domain are indicated. These pockets correspond to invaginations into the protein from the cytoplasmic side and are visualized here in a cut-away view. Black dot represents bound $\mathrm{Mg}^{2+}$.

Whereas the structure of AfAPT1 was solved only in the apo state, crystal structures of both the apo- and ligand-bound forms of AfAPT2 were reported. These highlight the important residues involved in ligand binding and catalysis (Figure 4). Although the fold and domain architecture of AfAPT2 is similar to that of AfAPT1, the presence of bound ligands gives us a better understanding of the catalytic mechanism. The structures (PDB $406 \mathrm{M}$ and $406 \mathrm{~N}$ ) directly shed light on the catalytic mechanism by assigning functions to the residues of the signature consensus motif of the CDP-alcohol phosphotransferase family of proteins. Briefly, residues D214, D217 and D235 (Figure 4) coordinate the pyrophosphate of CDP whereas D239 plays an important role in catalysis. D239 does not participate in binding to CDP but is located between the acceptor and donor binding sites, indicating its crucial role in catalysis. This residue facilitates nucleophilic attack of the 
activated acceptor on the $\beta$-phosphorus of the CDP, resulting in formation of CMP and a phosphodiester product. The mechanism seems universal for all members of the CDPalcohol phosphotransferase family.
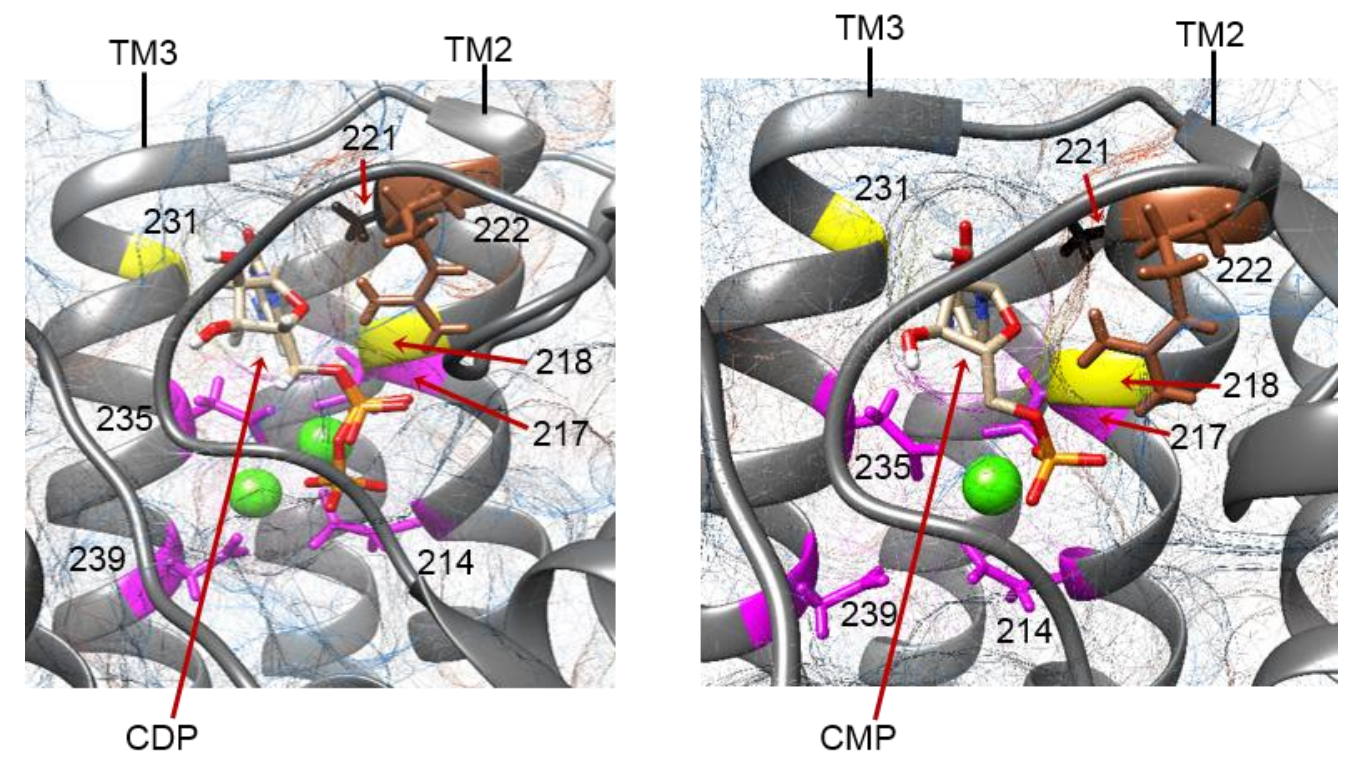

Figure 4. Ligand-bound forms of AfAPT2. Left and right panels depict the CDP-and CMP-bound forms of AfAPT2 with respect to the conserved consensus motifformed by residues of transmembrane domains 2 and 3. Aspartate (D), glycine (G), alanine (A) and arginine (R) residues forming the $\mathrm{D}(\mathrm{x})_{2} \mathrm{DG}(\mathrm{x})_{2} \mathrm{AR}(\mathrm{x})_{7-12} \mathrm{G}(\mathrm{x})_{3} \mathrm{D}(\mathrm{x})_{3} \mathrm{D}$ motifare shown in magenta, yellow, black and sienna sticks, respectively. The protein structure is shown as gray ribbon with the surface colored according to the hydrophobic scale. $\mathrm{Ca}^{2+}$ ions are shown as green spheres.

The structures of AfAPT1 and AfAPT2 reinforce many structure-function aspects of the CDP-alcohol phosphotransferase protein family that were previously known only through mutagenesis and bioinformatics studies, and illuminate the enzymatic mechanism. Importantly, the membrane topology of the DIPPS domain and its cytoplasmically exposed substrate-binding pocket for CDP-alcohol reinforces the prevailing view of the topology of phospholipid reactions in the ER (Figure 2).

\section{Bilayer assembly requires lipid scrambling}

The problem of how newly synthesized phospholipids are translocated (flipped) from the cytoplasmic to the luminal side of the ER remains unsolved, despite the passage of more 
than 35 years since such translocation events were first measured in biogenic membranes (for example [29]). It is clear that such a translocation event is necessary in order to deposit newly synthesized lipids into the luminal leaflet of the ER membrane bilayer (Figure 2) and ensure uniform expansion of the bilayer during cell growth [18]. As spontaneous transbilayer movement of phospholipids occurs infrequently because of the energetic challenge of reorienting the lipid headgroup through the hydrophobic interior of the bilayer, a cost of $>20 \mathrm{kcal} / \mathrm{mole}$, the ER must have specific mechanisms to accelerate the rate of lipid flipping [18]. This is indeed the case as revealed by a number of studies on phospholipid translocation using ER-derived microsomal vesicles and also synthetic proteoliposomes reconstituted with ER proteins [26,30-35]. All the studies point to a role for a specific membrane protein - a phospholipid scramblase - in mediating constitutive, bidirectional, ATP-independent transbilayer movement of common phospholipids across the ER membrane. Interestingly, measured rates of phospholipid scrambling are much higher than needed for cell growth $[18,36]$, consistent with a constitutive activity, while specificity is relatively low as non-natural structural isomers of glycerophospholipids (for example lipids with an sn-2,3-diacylglycerol moiety instead of the $s n$-1,2-diacylglycerol found in eukaryotes [33]) as well as ceramide-based lipids such as sphingomyelin and glucosylceramide are translocated equally well [37]. However, some lipids such as the isoprenoid-based glycolipids involved in the biosynthesis of protein $N$-glycans are not translocated by phospholipid scramblases [35]. Reconstitution studies indicate that scramblase activity can be enriched in specific fractions of detergent-solubilized ER membrane proteins. Thus, analysis of Triton X-100-solubilized ER membrane proteins by velocity gradient sedimentation reveals that scramblase activity is associated with proteins that sediment at $\sim 4 S$ [33-35] and that bind dye resins from which they can be eluted in a sharp profile with low salt concentrations [35].

Although the first biochemical reconstitution of ER phospholipid scramblase activity was reported in 1987 [32], the ER phospholipid scramblase has yet to be identified at a molecular level. Why has progress in this area been so slow? Biochemical approaches to this problem are traditionally considered difficult as membrane proteins are involved, and the handling of lipids and the measurement of their transport represent unusual technical challenges. More importantly, it has been suggested that scramblase activity may be due to 
several proteins functioning independently, making the identification of a single scramblase protein via a genetic or biochemical reconstitution approach potentially difficult. An extension of this idea is that all proteins have some scramblase activity. Although this notion is supported by reports of the ability of certain transmembrane peptides to promote scrambling at a low rate [38,39], it is unlikely to represent a major mechanism as biochemical fractionation of Triton X-100-solubilized ER membrane proteins clearly identifies large cohorts of ER proteins that have no scramblase activity [35]. Further possibilities are that poorly folded membrane proteins destined for degradation via ER quality control pathways may disturb the bilayer, inducing defects that promote scrambling: such proteins may individually be poor scramblases, but collectively they might provide sufficient 'background' activity to overcome deficiencies in dedicated scramblases. While this idea is interesting, it conflicts with the experimental result that orthogonal fractionation approaches (discussed above) are able to enrich scramblase activity from a crude mixture of ER membrane proteins [35]. The fractionation studies strongly suggest that scramblase activity is either due to a single protein or due to a small group of proteins with properties that are so similar that the proteins co-fractionate even when distinct fractionation criteria are deployed.

Despite lack of progress on the ER scramblase, other scramblase proteins have nevertheless been identified in recent years. These discoveries represent major advances (Montigny et al. 2016, this special issue). It has been known for decades that the plasma membrane of eukaryotic cells has an asymmetric transbilayer lipid distribution with phosphatidylserine (PS) confined to the cytoplasmic leaflet. Regulated exposure of PS at the cell surface is physiologically very important $[40,41]$. Thus, cells undergoing apoptosis expose PS as a recognition signal, marking them for clearance by macrophages. Activated blood platelets expose PS to generate a procoagulant surface necessary for the synthesis of blood clotting factors. The mechanisms by which PS becomes exposed at the cell surface were mysterious, until recently, when members of the Xkr8 protein family were shown to be involved in PS exposure during apoptosis $[42,43]$, and TMEM16F, a member of the $\mathrm{Ca}^{2+}$ dependent TMEM16 family of ion channels, was shown to be required for PS exposure on activated platelets [19]. Whereas the scramblase activity of fungal members of the TMEM16 protein family has been demonstrated explicitly by reconstitution of purified 
proteins $[20,21]$ it remains to be seen whether the Xkr8 proteins are indeed scramblases or whether they are indirect contributors to PS exposure during apoptosis.

More than 20 years ago it was reported that the disc membranes of rod photoreceptor cells of the retina have an ER-like phospholipid scramblase activity: phospholipid analogues were translocated unspecifically across discs by a bidirectional, ATPindependent mechanism [24,44,45]. Unexpectedly, the light-sensing G protein-coupled receptor (GPCR) rhodopsin was identified as the disc phospholipid scramblase [22-24]. Other Class A GPCRs, including the $\beta 1$ - and $\beta 2$-adrenergic receptors, turned out to have scramblase activity as well $[22,23]$. Rhodopsin's scramblase activity is implicated in aspects of photoreceptor disc membrane homeostasis [24] whereas the physiological function of the scramblase activity of other GPCRs remains to be determined. GPCRs are cell surface proteins and unlikely to be constitutively active at the plasma membrane as such activity would likely eliminate lipid asymmetry and inappropriately position PS at the cell surface; it has been speculated - but remains to be tested - that GPCR scramblase activity may be silenced by the high cholesterol content of the plasma membrane $[23,24]$. Like all proteins destined for the cell surface, GPCRs are first synthesized and integrated into the ER membrane before entering secretory vesicles for export. It is interesting to speculate that a transiting pool of GPCRs may provide the necessary scramblase activity for ER membrane biogenesis [23]. If this is the case, it should be possible to co-enrich the relevant GPCRs via the orthogonal fractionation procedures discussed above.

\section{Activating the tail for lipid assembly: synthesis of the liponucleotide CDP- diacylglycerol for synthesis of ER and mitochondrial phospholipids}

We now turn to glycerophospholipid biosynthesis reactions where the high-energy CDPalcohol precursor is the activated lipid tail CDP-diacylglycerol (CDP-DAG). These reactions synthesize PI, phosphatidylglycerol (PG) and cardiolipin (CL) (Figure 1). PI is a constituent of most eukaryotic membranes, but also serves as precursor of a large and structurally complex group of membrane-bound signaling molecules located in different intracellular compartments (recently reviewed in [46]), as well as the synthesis of glycosylphosphatidylinositol (GPI) lipids and protein anchors in the plasma membrane 
([47], recently reviewed in [48,49]). In contrast, PG and CL are signature mitochondrial glycerophospholipids. CL has a unique dimeric structure consisting of two phosphatidyl moieties linked to glycerol and plays an important role in mediating the formation and maintenance of multi-subunit oxidative phosphorylation complexes and supercomplexes and their interaction with the ADP-ATP-carrier [50-53] (recently reviewed in [54-57]). Surprisingly, despite its roles in mitochondrial membrane protein complex formation and stabilization, CL synthesis in S. cerevisiae is not essential for growth [58,59]. In contrast, the synthesis of CL is essential for growth of T. brucei parasites [60].

CDP-DAG is produced from phosphatidic acid (PA) and CTP in a reaction catalyzed by the integral membrane protein CDP-DAG synthase (CDS)(Figure 1). A recent report described the crystal structure of CDS from the hyperthermophilic bacterium Thermotoga maritima [14], providing the first molecular-level insights into CDS since its activity was first described by Paulus and Kennedy in 1960 [8]. The protein (termed TmCdsA) was crystallized as a dimer, with each subunit showing a deep funnel-shaped cavity containing a catalytically important $\mathrm{Mg}^{2+}, \mathrm{K}^{+}$-hetero-di-metal center and open both to the cytoplasmic side of the bilayer and to the membrane, consistent with entry and exit of the water-soluble and lipidic substrates (CTP, PA) and products (pyrophosphate, CDP-DAG) (Figure 5). PA could enter the reaction cavity from either the inner (cytoplasmic) or outer leaflet of the membrane; both possibilities are depicted in Figure 4. The CDP-DAG product of the reaction presumably exits on the cytoplasmic side. The catalytic mechanism appears to be analogous to the dual-ion mechanism proposed for enzymes that make and break nucleic acids $[61,62]$. The first step is the destabilization of the $\alpha$ - $\beta$-phosphodiester bond of CTP and the second step is the transfer of the CMP to PA. The phosphate group of PA, activated by $\mathrm{Mg}^{2+}$, initiates a nucleophilic attack on the $\alpha$-phosphate of CTP. As a result the $\beta \gamma$ pyrophosphate moiety of CTP is released with the assistance of $\mathrm{K}^{+}$, followed by the transfer of CMP to PA. 
A
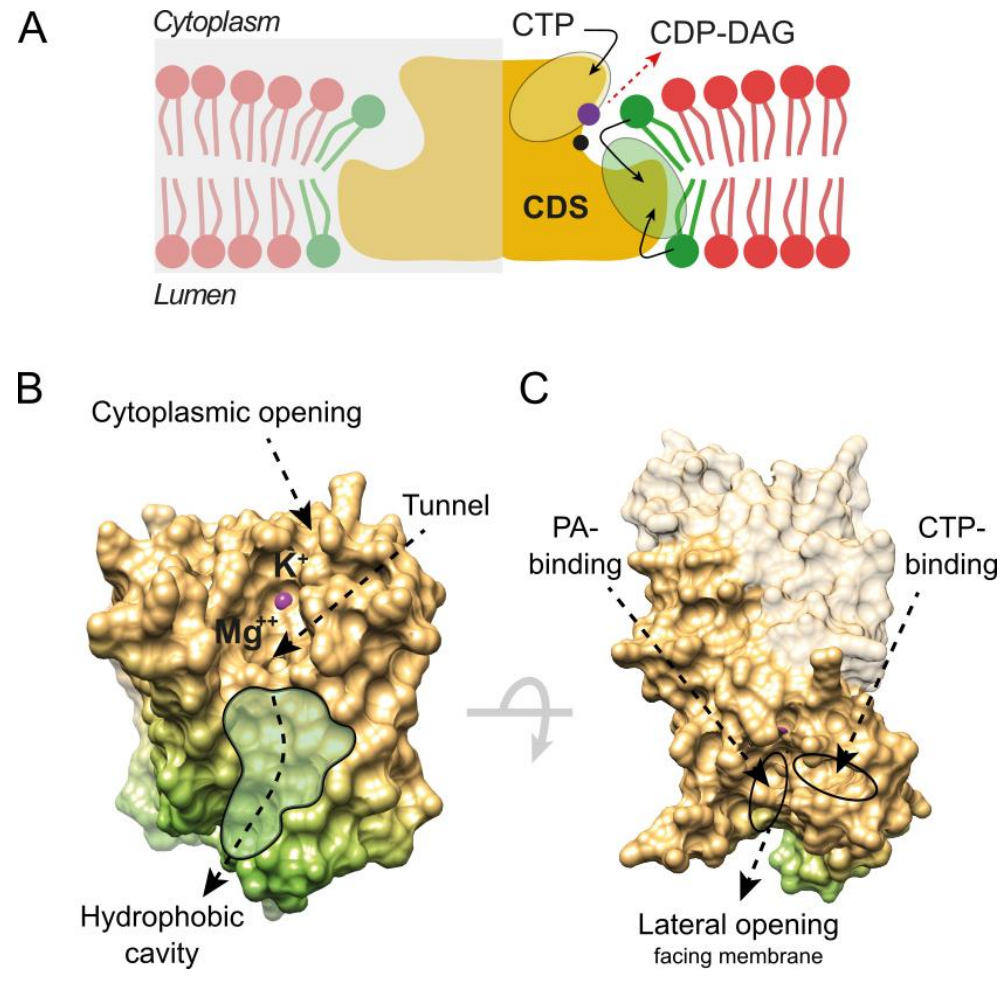

Figure 5. CDP-DAG synthase.

Schematic representation of CDPdiacylglycerol synthase (CDS) based on the crystal structure [14]. The protein is situated in a membrane bilayer (phospholipids shown in red). A, The hydrophilic ligand, CTP, binds to the hydrophilic pocket shown in light yellow, whereas the lipid substrate, PA (shown in green), enters the laterally open hydrophobic cavity (light green) from the membrane itself. Entry could be from either the inner (cytoplasmic) or outer leaflet - both options are shown. The lipid product CDP-DAG is

then released into the cytoplasmic leaflet. The black and purple dots represent $\mathrm{Mg}^{2+}$ and $\mathrm{K}^{+}$, respectively. B, Crystal structure of CDS showing the $\mathrm{Mg}^{2+} / \mathrm{K}^{+}$di-metal center and the hydrophobic cavity for PA. C, Side view of CDS showing PA- and CTP-binding sites. The second unit of the dimer is made translucent for clarity.

Early biochemical studies in yeast and mammalian cells showed that CDS activity is enriched in microsomal/ER fractions and the inner mitochondrial membrane [63-66]. In yeast CDS is encoded by a single gene, $C D S 1$ [67]. Although the precise localization of $S$. cerevisiae Cds1 was not determined, it was generally believed that synthesis of CDP-DAG occurs in the ER, where it is used for PI and PS formation [68]. However, since CDP-DAG is also required for mitochondrial PG and CL synthesis, it was unclear if CDS has a dual localization or if CDP-DAG is transported from its site of synthesis in the ER to its site of usage in mitochondria. Only recently, detailed localization studies in yeast revealed that $S$. cerevisiae Cds1 is exclusively present in the ER [69]. Interestingly, in the same report, a novel mitochondrial CDS, Tam41, was identified [69]. Tam41 is a peripheral protein associating with the matrix side of the inner mitochondrial membrane. It was originally identified as a maintenance protein for the Tim23 complex $[70,71]$ and was found to be 
involved in CL formation [72]. The identification of Tam41 in the inner mitochondrial membrane demonstrated that local production of CDP-DAG for PG and CL synthesis is available, making putative transport pathways for CDP-DAG unnecessary. Interestingly, over-expression of ER-localized Cds1 was unable to restore CL levels in tam $41 \Delta$ cells, indicating that transport of CDP-DAG from the ER to mitochondria is inefficient [69].

In mammals, two isoforms of CDS have been described, CDS1 and CDS2 [73,74]. The enzymes show different substrate specificities [75], however, both forms seem to be localized in the ER [75-77]. In addition, the human genome contains a homolog of yeast tam41, suggesting that besides CDS1/2, mammals may also express a mitochondrial CDS. It is tempting to speculate that CDS1/2 and TAM41 may reflect the chromatographically distinct CDP-DAG synthases described more than 20 years ago in microsomal and mitochondrial fractions, respectively, from liver cells $[65,78]$.

The active site of Tam41 in S. cerevisiae faces the matrix [69]. This is consistent with the proposed localization of the active sites of the enzymes involved in the subsequent synthesis of PG and CL (reviewed in [79]). The discovery of a mitochondrial CDS solved a long-standing issue about the sub-cellular distribution of the enzyme and/or the delivery of CDP-DAG to the mitochondrion. In addition, its topological orientation indicates that all reactions involved in the formation of $\mathrm{CL}$, with the exception of CL remodeling steps (reviewed in [79]), occur on the matrix side of the inner mitochondrial membrane.

\section{Synthesis of a phospholipid from CDP-DAG: structure of a bacterial homolog of phosphatidylinositol synthase}

The remarkable burst of structural information on lipid biosynthetic enzymes in the last couple of years includes the structure of a CDP-DAG-utilizing enzyme from the Grampositive bacterium R. salmoninarum [17]. This enzyme is a phosphatidylinositol-phosphate synthase (termed RsPIPS) that uses inositol-P and CDP-DAG as substrates. Its reaction is analogous to that of PI synthase (PIS), the eukaryotic enzyme that synthesizes PI from inositol and CDP-DAG and not to be confused with the kinase-mediated reactions that generate PI monophosphates from PI.

High-resolution structures of apo- and CDP-DAG-bound RsPIPS, in the form of fusion proteins, with the fusion partner corresponding to the cytoplasmic domain of AfAPT2 
(equivalent to the IPCT domain of AfAPT1 (Figure 3)) [17], reveal a homodimeric architecture. Each monomer comprises six transmembrane $\alpha$-helices, similar to the structures of the DIPPS domain of AfAPT1 [15] and the membrane domain of the CDPalcohol-phosphotransferase AfAPT2 [16]. The authors suggest that this architecture is conserved for all CDP-alcohol-phosphotransferases that use soluble acceptors such as inositol or inositol phosphate, regardless of the CDP-activated donor molecule involved. Thus CEPT, CPT and EPT (Figures 1 and 2), all of which use the lipidic acceptor DAG, would be expected to have additional transmembrane helices to accommodate the acyl chains of the acceptor.

RsPIPS has a hydrophilic cavity that can accommodate inositol-1-phosphate entering from the cytoplasmic side. The hydrophilic cavity extends to a hydrophobic cavity that harbors CDP-DAG. The hydrophobic cavity is open to the membrane, permitting lateral entry of CDP-DAG reminiscent of the entry of PA into the active site of CDS (Figure 6). This membrane-exposed cavity is not present in AfAPT1 (Figure 3) [15] that has only watersoluble substrates and products, nor does it exist in AfAPT2 (Figure 4) [16], which likewise probably only handles water-soluble molecules.

A

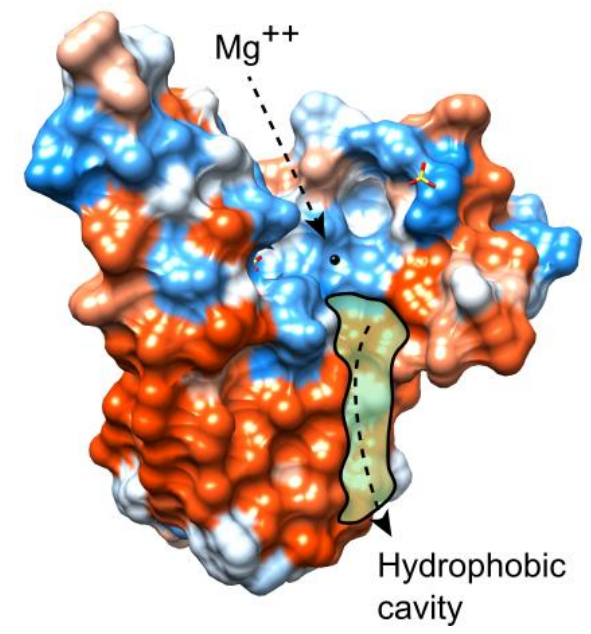

B

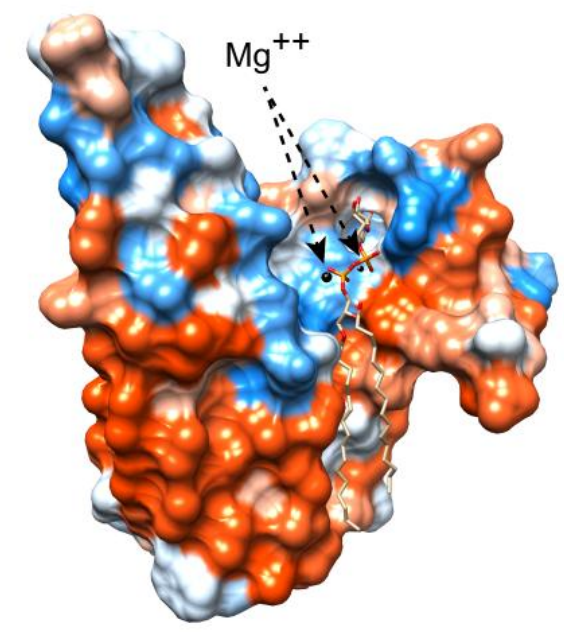

Figure 6. Two states of PIP-synthase, a CDPalcohol phosphotransferase. Apo- (A) and ligandbound ( $\boldsymbol{B}$ ) forms of RsPIPS are depicted in surface representations (the cytoplasmic domain used for crystallization is

excluded for clarity). The surface is color coded to reflect hydrophobicity (blue, hydrophilic and orange, hydrophobic). $\mathrm{Mg}^{2+}$ is shown as a black dot and the ligand (panel B) is shown in stick representation. The empty hydrophobic pocket (panel A) is highlighted by an irregular green shape. 


\section{Subcellular location and membrane topology of phosphatidylinositol synthesis in two unicellular model eukaryotes}

PI is synthesized from myo-inositol and CDP-DAG by PI synthases (equivalent to bacterial RsPIPS) belonging to the CDP-alcohol phosphotransferase superfamily. In $S$. cerevisiae, PI synthase (Pis1) activity has been found in the ER [64,80], mitochondria [64] and a sub-domain of the ER associated with mitochondria (mitochondria-associated membranes, or MAMs] [81]. Early subcellular fractionation and reconstitution experiments suggested that the active site of Pis1 may face the cytosol [64,82]. Very recently, by introducing cysteine residues at various positions in the primary sequence of $S$. cerevisiae Pis1 and monitoring their accessibility to alkylating reagents, the cytosolic location of the active site was confirmed [83] (Figure 7). In addition, an active site facing the cytosol is in line with modeling studies of Pis1 based on the crystal structures of $A$. fulgidus CDPalcohol-phosphotransferases (see above) [83,84]. In mammalian cells, PI synthase was originally found in a mitochondrial fraction from kidney cells [85] and the ER in rat lung pneumocytes [86,87]. More recently, in an attempt to determine the subcellular pools of PI and their functional significance, tagged forms of PIS and CDS were expressed in HEK and COS-7 cells [88]. Interestingly, the study revealed a highly mobile compartment of ER origin, which could be separated from bulk ER, as the main site of PI synthesis and distribution. Although the topology of the active site of mammalian PIS has not been determined, it is generally assumed that it faces the cytosol.

In contrast, in protozoan parasites the active site of PI synthase may be localized in the lumen of the ER and Golgi. In T. brucei, de novo synthesis of PI is essential for parasite growth in culture $[89,90]$. Interestingly however, inhibition of myo-inositol synthesis by conditionally knocking out the rate-limiting enzyme myo-inositol-3-phosphate synthase had no effect on cellular PI levels, instead it blocked the synthesis of GPI lipids and protein anchors [91]. Subsequent studies revealed that the synthesis of bulk PI is dependent on carrier-mediated uptake of myo-inositol from the environment [92,93]. The transporter, $T$. brucei $\mathrm{H}^{+}$-linked myo-inositol transporter (TbHMIT), was found to localize in the plasma membrane and the Golgi, indicating that exogenous myo-inositol is transported into the lumen of the Golgi for bulk PI synthesis. These results imply that the active site of PI synthase faces the luminal side of the Golgi membrane (Figure 7). In addition, they indicate 
that cellular PI is organized in two functionally different pools, one derived from de novo synthesized myo-inositol while the other uses exogenous myo-inositol for PI synthesis $[92,93]$. Interestingly, the existence of functionally different pools of PI in trypanosomes had been suggested more than 25 years ago. In a cell-free microsomal system capable of synthesizing GPI precursor lipids using exogenously added substrates, no incorporation of myo- $\left[{ }^{3} \mathrm{H}\right]$ inositol into GPI was observed, although the membranes were capable of [ $\left.{ }^{3} \mathrm{H}\right] \mathrm{PI}$ synthesis [94]. These results suggested that the PI pool used for GPI synthesis was different from the $m y o-\left[{ }^{3} \mathrm{H}\right]$ inositol-labeled PI pool and led the authors to speculate that PI may be synthesized in the lumen of the ER [94]. The recent identification of the T. brucei TbHMIT in the Golgi $[92,93]$ may explain these early findings. In addition, the dependence of GPI synthesis from de novo synthesized but not imported myo-inositol has recently also been observed in Plasmodium parasites [95].

Further evidence that the topology of yeast Pis1 is different from that of its trypanosome homolog can be deduced from (early) GPI labeling experiments. Traditionally, exogenous myo-[3H]inositol has been an excellent substrate to label GPI-anchored proteins in S. cerevisiae [96,97] but a very poor substrate in T. brucei cultures (see e.g. [91], and P. Bütikofer, A.K. Menon, unpublished data). In retrospective, these observations can now be explained by the sub-cellular localization and topology of PI synthase in these organisms (Figure 7): the active site of ER-bound yeast Pis1 faces the cytosol and, thus, can incorporate $m y o-\left[{ }^{3} \mathrm{H}\right]$ inositol taken up from the environment as substrate for GPI synthesis in the ER. In contrast, T. brucei PI synthase is localized in both the ER and Golgi [83], with the active site facing the lumen. The ER enzyme provides PI for GPI synthesis, using de novo synthesized myo-inositol, whereas the Golgi enzyme uses exogenous myo-[3H]inositol for bulk PI, but not GPI, synthesis after its TbHMIT-mediated transport into the lumen of the Golgi. Interestingly, the Golgi localization of a mammalian HMIT in rat primary cultured neurons [98] suggests that luminally-oriented PI synthesis in the Golgi may not be unique to protozoa but also be present in higher eukaryotes. 
Lipid topogenesis - 35 years on

A

S. cerevisiae T. brucei

Phosphatidic acid

$\downarrow$ CDP-DAG synthase

CDP-diacylglycerol

$\downarrow$ PI synthase

Phosphatidylinositol

Phosphatidic acid

CDP-diacylglycerol

Pl synthase

Phosphatidylinositol

Cytosol ER lumen Golgi lumen

$\mathrm{B}$

S. cerevisiae

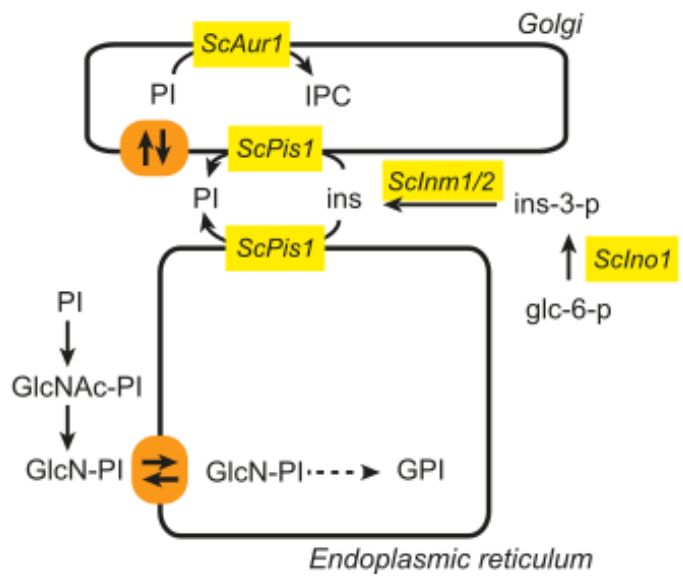

T. brucei

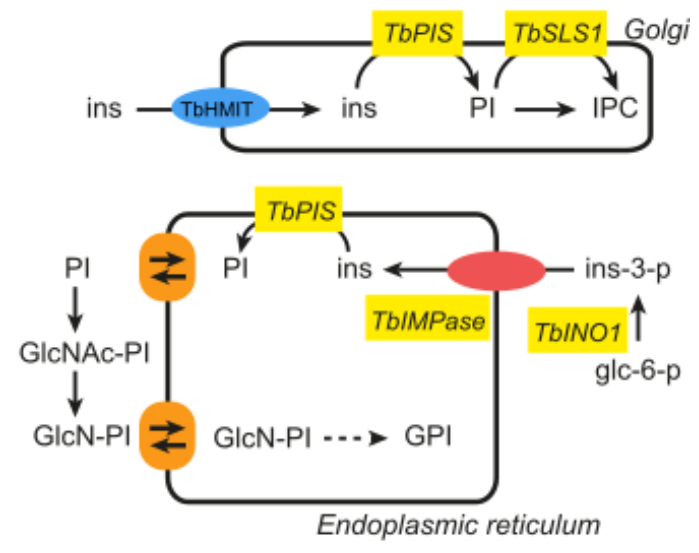

Figure 7. PI synthesis in two unicellular model eukaryotes. A, The orientation of the active site of PI synthase differs between yeast and trypanosomes. In S. cerevisiae, the catalytic domain is oriented towards the cytosol while it faces the lumen of the ER/Golgi in T. brucei. B, Schematic representation of the subcellular distribution of PI and GPI synthesis in the respective eukaryotes. PI is synthesized from CDP-DAG and myo-inositol. The latter can be obtained via de novo synthesis starting from glucose-6-phosphate in a conserved two-step reaction sequence catalyzed by 1-D-myo-inositol-3phosphate synthase and myo-inositol-monophosphatase [99]. Alternatively, or in addition, myoinositol can be taken up from the environment via sodium- or proton-linked myo-inositol symporters (in blue) [100-102]. Putative PI scramblases are in orange, a putative inositol-3-phosphate transporter is in red. glc-6-p, glucose-6-phosphate; GlcN, glucosamine; GlcNAc, N-acetylglucosamine; GPI, glycosylphosphatidylinositol; ins, myo-inositol; ins-3-p, inositol-3-phosphate; IPC, inositol phosphorylceramide; PI, phosphatidylinositol; ScAur1, S. cerevisiae inositol phosphorylceramide synthase; ScPis1, S. cerevisiae phosphatidylinositol synthase; ScInm1/2, S. cerevisiae inositol 
Lipid topogenesis - 35 years on

monophosphatase1/2; ScIno1, S. cerevisiae inositol-3-phosphate synthase; TbHMIT, T. brucei H+-linked myo-inositol transporter; TbPIS, T. brucei phosphatidylinositol synthase; TbINO1, T. brucei inositol monophosphatase; TbSLS1, T. brucei sphingolipid synthase 1; TbIMPase, T. brucei inositol monophosphatase.

\section{Conclusion}

What have we learned about early events of lipid topogenesis since the publication of the article by Bell et al. in 1981? The principle advances have been very recent. The molecular identities of phospholipid scramblases that operate in non-biogenic membranes such as the plasma membrane and disc membranes of photoreceptor cells were revealed in reports published during 2010-2013, and the first crystal structures of lipid biosynthetic enzymes were reported in 2014-2015. These advances provide new opportunities even as many questions remain. Discovering the identity of the ER phospholipid scramblase continues to be a challenge for future work. While structure-function information on the newly identified TMEM16 and GPCR scramblases will inevitably impact the search for the ER scramblase, it is worth considering whether these proteins, especially GPCRs, provide the ER with sufficient scramblase activity when they are integrated into the ER membrane prior to export to their final destination?

The new crystal structures of the CDP-alcohol phosphotransferases provide an obvious starting point to explore mechanistic questions about lipid biosynthesis. For example, how do lipid biosynthetic enzymes handle both lipid and water-soluble substrates? What are the routes for substrate entry and product exit? Other questions pertain to the site and topology of phospholipid synthesis. For example, while the standard topological model of phospholipid synthesis (Figure 2) is generally correct, the discovery that PI synthesis can occur in more than one subcellular compartment and with either luminal or cytoplasmic topology to generate distinct pools of PI reveals an unexpected complexity that needs to be further explored. How are these distinct pools of PI homeostatically controlled? How are they fed into pathways that generate PI-derived signaling molecules? Similarly, in most eukaryotes, PC and PE are synthesized in bulk by CEPT, CPT and EPT enzymes, but detailed information on their precise sub-cellular localization, and thus the site(s) of PC and PE synthesis, is scarce. While in yeast and mammalian cells CPT has been identified in the 
Golgi [25,103], mammalian and parasite EPT and CEPT enzymes have been reported in the ER/microsomal fractions [25,104-107]. Very recently, EPT in T. brucei was shown to localize exclusively to the perinuclear ER, whereas CEPT was distributed throughout the bulk ER [108]. Together these results indicate that the sites of PC and PE synthesis in eukaryotes may localize to different (sub-) compartments. In addition, since trypanosome EPT uses alkylacyl-glycerol, which is made in glycosomes, for ether-type PE formation, this raises the interesting question how the substrate is trafficked to the perinuclear ER.

\section{Acknowledgements}

We acknowledge support from National Institutes of Health (NIH) grants EY024207 and GM106717 (AKM), Swiss National Science Foundation (SNF) grant 149353 and an SNF international short visit stipend (PB), and Velux Stiftung grant no. 881 (PB, AKM). AKM

thanks Kathleen Battle, Sam Canis and Josef Myslivecek, and PB thanks Garry Moore and Stevie R. Vaughan for stimulation. 
Lipid topogenesis - 35 years on

\section{References}

[1] R.M. Bell, L.M. Ballas, R.A. Coleman, Lipid topogenesis, J. Lipid Res. 22 (1981) 391403.

[2] G. Blobel, Intracellular protein topogenesis., Proc. Natl. Acad. Sci. U. S. A. 77 (1980) 1496-500.

[3] J.E. Rothman, J. Lenard, Membrane asymmetry., Science. 195 (1977) 743-53.

[4] W. Dowhan, Molecular basis for membrane phospholipid diversity: why are there so many lipids?, Annu Rev Biochem. 66 (1997) 199-232.

doi:10.1146/annurev.biochem.66.1.199.

[5] C. Klose, M.A. Surma, K. Simons, Organellar lipidomics--background and perspectives., Curr. Opin. Cell Biol. 25 (2013) 406-13. doi:10.1016/j.ceb.2013.03.005.

[6] J.C.M. Holthuis, A.K. Menon, Lipid landscapes and pipelines in membrane homeostasis., Nature. 510 (2014) 48-57. doi:10.1038/nature13474.

[7] E.P. Kennedy, S.B. Weiss, The function of cytidine coenzymes in the biosynthesis of phospholipides, J Biol Chem. 222 (1956) 193-214.

[8] H. Paulus, E.P. Kennedy, The enzymatic synthesis of inositol monophosphatide., J. Biol. Chem. 235 (1960) 1303-11.

[9] G. Blobel, Protein targeting (Nobel lecture)., Chembiochem. 1 (2000) 86-102.

[10] J.E. Rothman, The principle of membrane fusion in the cell (Nobel lecture)., Angew. Chem. Int. Ed. Engl. 53 (2014) 12676-94. doi:10.1002/anie.201402380.

[11] A. Shevchenko, K. Simons, Lipidomics: coming to grips with lipid diversity., Nat. Rev. Mol. Cell Biol. 11 (2010) 593-8. doi:10.1038/nrm2934.

[12] B. Brügger, Lipidomics: analysis of the lipid composition of cells and subcellular organelles by electrospray ionization mass spectrometry., Annu. Rev. Biochem. 83 (2014) 79-98. doi:10.1146/annurev-biochem-060713-035324.

[13] G.R. V Hammond, T. Balla, Polyphosphoinositide binding domains: Key to inositol lipid biology., Biochim. Biophys. Acta. 1851 (2015) 746-58. doi:10.1016/j.bbalip.2015.02.013.

[14] X. Liu, Y. Yin, J. Wu, Z. Liu, Structure and mechanism of an intramembrane liponucleotide synthetase central for phospholipid biosynthesis., Nat. Commun. 5 
(2014) 4244. doi:10.1038/ncomms5244.

[15] P. Nogly, I. Gushchin, A. Remeeva, A.M. Esteves, N. Borges, P. Ma, et al., X-ray structure of a CDP-alcohol phosphatidyltransferase membrane enzyme and insights into its catalytic mechanism., Nat. Commun. 5 (2014) 4169. doi:10.1038/ncomms5169.

[16] G. Sciara, O.B. Clarke, D. Tomasek, B. Kloss, S. Tabuso, R. Byfield, et al., Structural basis for catalysis in a CDP-alcohol phosphotransferase., Nat. Commun. 5 (2014) 4068. doi:10.1038/ncomms5068.

[17] O.B. Clarke, D. Tomasek, C.D. Jorge, M.B. Dufrisne, M. Kim, S. Banerjee, et al., Structural basis for phosphatidylinositol-phosphate biosynthesis., Nat. Commun. 6 (2015) 8505. doi:10.1038/ncomms9505.

[18] S. Sanyal, A.K. Menon, Flipping lipids: why an' what's the reason for?, ACS Chem. Biol. 4 (2009) 895-909. doi:10.1021/cb900163d.

[19] J. Suzuki, M. Umeda, P.J. Sims, S. Nagata, Calcium-dependent phospholipid scrambling by TMEM16F., Nature. 468 (2010) 834-8. doi:10.1038/nature09583.

[20] M. Malvezzi, M. Chalat, R. Janjusevic, A. Picollo, H. Terashima, A.K. Menon, et al., Ca2+dependent phospholipid scrambling by a reconstituted TMEM16 ion channel., Nat. Commun. 4 (2013) 2367. doi:10.1038/ncomms3367.

[21] J.D. Brunner, N.K. Lim, S. Schenck, A. Duerst, R. Dutzler, X-ray structure of a calciumactivated TMEM16 lipid scramblase., Nature. 516 (2014) 207-12. doi:10.1038/nature13984.

[22] I. Menon, T. Huber, S. Sanyal, S. Banerjee, P. Barré, S. Canis, et al., Opsin is a phospholipid flippase., Curr. Biol. 21 (2011) 149-53. doi:10.1016/j.cub.2010.12.031.

[23] M.A. Goren, T. Morizumi, I. Menon, J.S. Joseph, J.S. Dittman, V. Cherezov, et al., Constitutive phospholipid scramblase activity of a G protein-coupled receptor., Nat. Commun. 5 (2014) 5115. doi:10.1038/ncomms6115.

[24] O.P. Ernst, A.K. Menon, Phospholipid scrambling by rhodopsin., Photochem. Photobiol. Sci. 14 (2015) 1922-31. doi:10.1039/c5pp00195a.

[25] A.L. Henneberry, M.M. Wright, C.R. McMaster, The major sites of cellular phospholipid synthesis and molecular determinants of fatty acid and lipid head group specificity, Mol Biol Cell. 13 (2002) 3148-3161. doi:10.1091/mbc.01-11-0540.

[26] A.K. Menon, Watkins WE3rd, S. Hrafnsdóttir, Specific proteins are required to 
translocate phosphatidylcholine bidirectionally across the endoplasmic reticulum., Curr. Biol. 10 (2000) 241-52.

[27] M. V Rodrigues, N. Borges, M. Henriques, P. Lamosa, R. Ventura, C. Fernandes, et al., Bifunctional CTP:inositol-1-phosphate cytidylyltransferase/CDP-inositol:inositol-1phosphate transferase, the key enzyme for di-myo-inositol-phosphate synthesis in several (hyper)thermophiles., J. Bacteriol. 189 (2007) 5405-12. doi:10.1128/JB.00465-07.

[28] L.G. Gonçalves, N. Borges, F. Serra, P.L. Fernandes, H. Dopazo, H. Santos, Evolution of the biosynthesis of di-myo-inositol phosphate, a marker of adaptation to hot marine environments., Environ. Microbiol. 14 (2012) 691-701. doi:10.1111/j.14622920.2011.02621.x.

[29] J.E. Rothman, E.P. Kennedy, Rapid transmembrane movement of newly synthesized phospholipids during membrane assembly., Proc. Natl. Acad. Sci. U. S. A. 74 (1977) 1821-5.

[30] W.R. Bishop, R.M. Bell, Assembly of the endoplasmic reticulum phospholipid bilayer: the phosphatidylcholine transporter., Cell. 42 (1985) 51-60.

[31] X. Buton, G. Morrot, P. Fellmann, M. Seigneuret, Ultrafast glycerophospholipidselective transbilayer motion mediated by a protein in the endoplasmic reticulum membrane., J. Biol. Chem. 271 (1996) 6651-7.

[32] J.M. Backer, E.A. Dawidowicz, Reconstitution of a phospholipid flippase from rat liver microsomes., Nature. 327 (n.d.) 341-3. doi:10.1038/327341a0.

[33] R.A. Vishwakarma, S. Vehring, A. Mehta, A. Sinha, T. Pomorski, A. Herrmann, et al., New fluorescent probes reveal that flippase-mediated flip-flop of phosphatidylinositol across the endoplasmic reticulum membrane does not depend on the stereochemistry of the lipid., Org. Biomol. Chem. 3 (2005) 1275-83. doi:10.1039/b500300h.

[34] S. Vehring, L. Pakkiri, A. Schröer, N. Alder-Baerens, A. Herrmann, A.K. Menon, et al., Flip-flop of fluorescently labeled phospholipids in proteoliposomes reconstituted with Saccharomyces cerevisiae microsomal proteins., Eukaryot. Cell. 6 (2007) 162534. doi:10.1128/EC.00198-07.

[35] S. Sanyal, C.G. Frank, A.K. Menon, Distinct flippases translocate glycerophospholipids 
and oligosaccharide diphosphate dolichols across the endoplasmic reticulum., Biochemistry. 47 (2008) 7937-46. doi:10.1021/bi800723n.

[36] S.N. Gummadi, A.K. Menon, Transbilayer movement of dipalmitoylphosphatidylcholine in proteoliposomes reconstituted from detergent extracts of endoplasmic reticulum. Kinetics of transbilayer transport mediated by a single flippase and identification of protein fractions enriched in, J. Biol. Chem. 277 (2002) 25337-43. doi:10.1074/jbc.M203809200.

[37] M. Chalat, I. Menon, Z. Turan, A.K. Menon, Reconstitution of glucosylceramide flipflop across endoplasmic reticulum: implications for mechanism of glycosphingolipid biosynthesis., J. Biol. Chem. 287 (2012) 15523-32. doi:10.1074/jbc.M112.343038.

[38] M.A. Kol, A.I.P.M. de Kroon, J.A. Killian, B. de Kruijff, Transbilayer movement of phospholipids in biogenic membranes., Biochemistry. 43 (2004) 2673-81. doi:10.1021/bi036200f.

[39] M. Langer, R. Sah, A. Veser, M. Gütlich, D. Langosch, Structural properties of model phosphatidylcholine flippases., Chem. Biol. 20 (2013) 63-72. doi:10.1016/j.chembiol.2012.11.006.

[40] E.M. Bevers, P.L. Williamson, Phospholipid scramblase: an update., FEBS Lett. 584 (2010) 2724-30. doi:10.1016/j.febslet.2010.03.020.

[41] H.M. Hankins, R.D. Baldridge, P. Xu, T.R. Graham, Role of flippases, scramblases and transfer proteins in phosphatidylserine subcellular distribution., Traffic. 16 (2015) 35-47. doi:10.1111/tra.12233.

[42] J. Suzuki, D.P. Denning, E. Imanishi, H.R. Horvitz, S. Nagata, Xk-related protein 8 and CED-8 promote phosphatidylserine exposure in apoptotic cells., Science. 341 (2013) 403-6. doi:10.1126/science.1236758.

[43] K. Segawa, S. Nagata, An Apoptotic “Eat Me” Signal: Phosphatidylserine Exposure., Trends Cell Biol. 25 (2015) 639-50. doi:10.1016/j.tcb.2015.08.003.

[44] G. Wu, W.L. Hubbell, Phospholipid asymmetry and transmembrane diffusion in photoreceptor disc membranes., Biochemistry. 32 (1993) 879-88.

[45] E. Hessel, A. Herrmann, P. Müller, P.P. Schnetkamp, K.P. Hofmann, The transbilayer distribution of phospholipids in disc membranes is a dynamic equilibrium evidence for rapid flip and flop movement., Eur. J. Biochem. 267 (2000) 1473-83. 
[46] R.H. Michell, Inositol lipids: from an archaeal origin to phosphatidylinositol 3,5bisphosphate faults in human disease., FEBS J. 280 (2013) 6281-94. doi:10.1111/febs.12452.

[47] P. Orlean, A.K. Menon, Thematic review series: lipid posttranslational modifications. GPI anchoring of protein in yeast and mammalian cells, or: how we learned to stop worrying and love glycophospholipids, J Lipid Res. 48 (2007) 993-1011. doi:R700002-JLR200 [pii]10.1194/jlr.R700002-JLR200.

[48] M. Muñiz, C. Zurzolo, Sorting of GPI-anchored proteins from yeast to mammals-common pathways at different sites?, J. Cell Sci. 127 (2014) 2793-801. doi:10.1242/jcs.148056.

[49] T. Kinoshita, Biosynthesis and deficiencies of glycosylphosphatidylinositol., Proc. Jpn. Acad. Ser. B. Phys. Biol. Sci. 90 (2014) 130-43.

[50] M. Zhang, E. Mileykovskaya, W. Dowhan, Gluing the respiratory chain together. Cardiolipin is required for supercomplex formation in the inner mitochondrial membrane., J. Biol. Chem. 277 (2002) 43553-6. doi:10.1074/jbc.C200551200.

[51] K. Pfeiffer, V. Gohil, R.A. Stuart, C. Hunte, U. Brandt, M.L. Greenberg, et al., Cardiolipin Stabilizes Respiratory Chain Supercomplexes, J. Biol. Chem. 278 (2003) 5287352880. doi:10.1074/jbc.M308366200.

[52] M. Zhang, E. Mileykovskaya, W. Dowhan, Cardiolipin is essential for organization of complexes III and IV into a supercomplex in intact yeast mitochondria., J. Biol. Chem. 280 (2005) 29403-8. doi:10.1074/jbc.M504955200.

[53] S.M. Claypool, Y. Oktay, P. Boontheung, J.A. Loo, C.M. Koehler, Cardiolipin defines the interactome of the major ADP/ATP carrier protein of the mitochondrial inner membrane., J. Cell Biol. 182 (2008) 937-50. doi:10.1083/jcb.200801152.

[54] E. Mileykovskaya, W. Dowhan, Cardiolipin-dependent formation of mitochondrial respiratory supercomplexes., Chem. Phys. Lipids. 179 (2014) 42-8. doi:10.1016/j.chemphyslip.2013.10.012.

[55] G. Paradies, V. Paradies, V. De Benedictis, F.M. Ruggiero, G. Petrosillo, Functional role of cardiolipin in mitochondrial bioenergetics., Biochim. Biophys. Acta. 1837 (2014) 408-17. doi:10.1016/j.bbabio.2013.10.006.

[56] M. Ren, C.K.L. Phoon, M. Schlame, Metabolism and function of mitochondrial 
cardiolipin., Prog. Lipid Res. 55 (2014) 1-16. doi:10.1016/j.plipres.2014.04.001.

[57] V. Raja, M.L. Greenberg, The functions of cardiolipin in cellular metabolism-potential modifiers of the Barth syndrome phenotype., Chem. Phys. Lipids. 179 (2014) 49-56. doi:10.1016/j.chemphyslip.2013.12.009.

[58] F. Jiang, H.S. Rizavi, M.L. Greenberg, Cardiolipin is not essential for the growth of Saccharomyces cerevisiae on fermentable or non-fermentable carbon sources., Mol. Microbiol. 26 (1997) 481-91.

[59] S.C. Chang, P.N. Heacock, C.J. Clancey, W. Dowhan, The PEL1 gene (renamed PGS1) encodes the phosphatidylglycero-phosphate synthase of Saccharomyces cerevisiae., J. Biol. Chem. 273 (1998) 9829-36. doi:10.1074/jbc.273.16.9829.

[60] M. Serricchio, P. Bütikofer, An essential bacterial-type cardiolipin synthase mediates cardiolipin formation in a eukaryote, Proc Natl Acad Sci U S A. 109 (2012) E954-61. doi:1121528109 [pii] 10.1073/pnas.1121528109.

[61] T.A. Steitz, J.A. Steitz, A general two-metal-ion mechanism for catalytic RNA., Proc. Natl. Acad. Sci. U. S. A. 90 (1993) 6498-502.

[62] W. Yang, J.Y. Lee, M. Nowotny, Making and breaking nucleic acids: two-Mg2+-ion catalysis and substrate specificity., Mol. Cell. 22 (2006) 5-13. doi:10.1016/j.molcel.2006.03.013.

[63] H.H. Bishop, K.P. Strickland, Studies on the formation by rat brain preparations of CDP-diglyceride from CTP and phosphatidic acids of varying fatty acid compositions, Can. J. Biochem. 54 (1976) 249-260. doi:10.1139/o76-038.

[64] K. Kuchler, G. Daum, F. Paltauf, Subcellular and submitochondrial localization of phospholipid-synthesizing enzymes in Saccharomyces cerevisiae., J. Bacteriol. 165 (1986) 901-10.

[65] A.Y.P. Mok, G.E. McDougall, W.C. McMurray, CDP-diacylglycerol synthesis in rat liver mitochondria, FEBS Lett. 312 (1992) 236-240. doi:10.1016/0014-5793(92)80942-A.

[66] M.J. Kelley, G.M. Carman, Purification and characterization of CDP-diacylglycerol synthase from Saccharomyces cerevisiae., J. Biol. Chem. 262 (1987) 14563-70.

[67] H. Shen, P.N. Heacock, C.J. Clancey, W. Dowhan, The CDS1 Gene Encoding CDPdiacylglycerol Synthase In Saccharomyces cerevisiae Is Essential for Cell Growth, J. Biol. Chem. 271 (1996) 789-795. doi:10.1074/jbc.271.2.789. 
[68] G.M. Carman, J. Matas, Solubilization of microsomal-associated phosphatidylserine synthase and phosphatidylinositol synthase from Saccharomyces cerevisiae., Can. J. Microbiol. 27 (1981) 1140-9.

[69] Y. Tamura, Y. Harada, S. Nishikawa, K. Yamano, M. Kamiya, T. Shiota, et al., Tam41 is a CDP-diacylglycerol synthase required for cardiolipin biosynthesis in mitochondria., Cell Metab. 17 (2013) 709-18. doi:10.1016/j.cmet.2013.03.018.

[70] Y. Tamura, Y. Harada, K. Yamano, K. Watanabe, D. Ishikawa, C. Ohshima, et al., Identification of Tam41 maintaining integrity of the TIM23 protein translocator complex in mitochondria., J. Cell Biol. 174 (2006) 631-7. doi:10.1083/jcb.200603087.

[71] M.R. Gallas, M.K. Dienhart, R.A. Stuart, R.M. Long, Characterization of Mmp37p, a Saccharomyces cerevisiae mitochondrial matrix protein with a role in mitochondrial protein import., Mol. Biol. Cell. 17 (2006) 4051-62. doi:10.1091/mbc.E06-04-0366.

[72] S. Kutik, M. Rissler, X.L. Guan, B. Guiard, G. Shui, N. Gebert, et al., The translocator maintenance protein Tam41 is required for mitochondrial cardiolipin biosynthesis., J. Cell Biol. 183 (2008) 1213-21. doi:10.1083/jcb.200806048.

[73] A.M. Heacock, M.D. Uhler, B.W. Agranoff, Cloning of CDP-diacylglycerol synthase from a human neuronal cell line., J. Neurochem. 67 (2002) 2200-2203. doi:10.1046/j.1471-4159.1996.67052200.x.

[74] M. Volta, A. Bulfone, C. Gattuso, E. Rossi, M. Mariani, G.G. Consalez, et al., Identification and Characterization of CDS2, a Mammalian Homolog of theDrosophilaCDP-diacylglycerol Synthase Gene, Genomics. 55 (1999) 68-77. doi:10.1006/geno.1998.5610.

[75] K. D’Souza, Y.J. Kim, T. Balla, R.M. Epand, Distinct Properties of the Two Isoforms of CDP-Diacylglycerol Synthase, Biochemistry. 53 (2014) 7358-7367. doi:10.1021/bi501250m.

[76] S. Saito, K. Goto, A. Tonosaki, H. Kondo, Gene Cloning and Characterization of CDPdiacylglycerol Synthase from Rat Brain, J. Biol. Chem. 272 (1997) 9503-9509. doi:10.1074/jbc.272.14.9503.

[77] S.L. Inglis-Broadgate, L. Ocaka, R. Banerjee, M. Gaasenbeek, J.P. Chapple, M.E. Cheetham, et al., Isolation and characterization of murine Cds (CDP-diacylglycerol synthase) 1 and 2, Gene. 356 (2005) 19-31. doi:10.1016/j.gene.2005.04.037. 
[78] A.Y.P. Mok, G.E. McDougall, W.C. McMurray, Comparative studies of CDPdiacylglycerol synthase in rat liver mitochondria and microsomes, Biochem. Cell Biol. 71 (1993) 183-189. doi:10.1139/o93-029.

[79] M.G. Baile, Y.-W. Lu, S.M. Claypool, The topology and regulation of cardiolipin biosynthesis and remodeling in yeast, Chem. Phys. Lipids. 179 (2014) 25-31. doi:10.1016/j.chemphyslip.2013.10.008.

[80] E. Zinser, C.D. Sperka-Gottlieb, E. V Fasch, S.D. Kohlwein, F. Paltauf, G. Daum, Phospholipid synthesis and lipid composition of subcellular membranes in the unicellular eukaryote Saccharomyces cerevisiae., J. Bacteriol. 173 (1991) 2026-34.

[81] B. Gaigg, R. Simbeni, C. Hrastnik, F. Paltauf, G. Daum, Characterization of a microsomal subfraction associated with mitochondria of the yeast, Saccharomyces cerevisiae. Involvement in synthesis and import of phospholipids into mitochondria, Biochim. Biophys. Acta - Biomembr. 1234 (1995) 214-20. doi:10.1016/00052736(94)00287-Y.

[82] A.S. Fischl, M.J. Homann, M.A. Poole, G.M. Carman, Phosphatidylinositol synthase from Saccharomyces cerevisiae. Reconstitution, characterization, and regulation of activity., J. Biol. Chem. 261 (1986) 3178-83.

[83] A. Bochud, A. Conzelmann, The active site of yeast phosphatidylinositol synthase Pis1 is facing the cytosol., Biochim. Biophys. Acta. 1851 (2015) 629-40. doi:10.1016/j.bbalip.2015.02.006.

[84] P. Nogly, I. Gushchin, A. Remeeva, A.M. Esteves, N. Borges, P. Ma, et al., X-ray structure of a CDP-alcohol phosphatidyltransferase membrane enzyme and insights into its catalytic mechanism, Nat. Commun. 5 (2014). doi:10.1038/ncomms5169.

[85] B.W. Agranoff, R.M. Bradley, R.O. Brady, The enzymatic synthesis of inositol phosphatide., J. Biol. Chem. 233 (1958) 1077-83.

[86] J.J. Batenburg, W. Klazinga, L.M. van Golde, Regulation and location of phosphatidylglycerol and phosphatidylinositol synthesis in type II cells isolated from fetal rat lung., Biochim. Biophys. Acta. 833 (1985) 17-24.

[87] J.E. Bleasdale, N.E. Tyler, J.M. Snyder, Subcellular sites of synthesis of phosphatidylglycerol and phosphatidylinositol in type II pneumonocytes., Lung. 163 (1985) 345-59. 
[88] Y.J. Kim, M.L. Guzman-Hernandez, T. Balla, A highly dynamic ER-derived phosphatidylinositol-synthesizing organelle supplies phosphoinositides to cellular membranes., Dev. Cell. 21 (2011) 813-24. doi:10.1016/j.devcel.2011.09.005.

[89] K.L. Martin, T.K. Smith, Phosphatidylinositol synthesis is essential in bloodstream form Trypanosoma brucei, Biochem J. 396 (2006) 287-295. doi:BJ20051825 [pii]10.1042/BJ20051825.

[90] K.L. Martin, T.K. Smith, The myo-inositol-1-phosphate synthase gene is essential in Trypanosoma brucei., Biochem. Soc. Trans. 33 (2005) 983-5. doi:10.1042/BST20050983.

[91] K.L. Martin, T.K. Smith, The glycosylphosphatidylinositol (GPI) biosynthetic pathway of bloodstream-form Trypanosoma brucei is dependent on the de novo synthesis of inositol, Mol Microbiol. 61 (2006) 89-105. doi:MMI5216 [pii] 10.1111/j.13652958.2006.05216.x.

[92] A. Gonzalez-Salgado, M.E. Steinmann, E. Greganova, M. Rauch, P. Mäser, E. Sigel, et al., myo-Inositol uptake is essential for bulk inositol phospholipid but not glycosylphosphatidylinositol synthesis in Trypanosoma brucei, J Biol Chem. 287 (2012) 13313-13323. doi:10.1074/jbc.M112.344812.

[93] A. González-Salgado, M. Steinmann, L.L. Major, E. Sigel, J.-L. Reymond, T.K. Smith, et al., Trypanosoma brucei bloodstream forms depend upon uptake of myo-inositol for Golgi phosphatidylinositol synthesis and normal cell growth, Eukaryot. Cell. 14 (2015) EC.00038-15. doi:10.1128/EC.00038-15.

[94] A. Menon, R. Schwarz, S. Mayor, G. Cross, Cell-free synthesis of glycosylphosphatidylinositol precursors for the glycolipid membrane anchor of Trypanosoma brucei variant surface glycoproteins. Structural characterization of putative biosynthetic intermediates, J. Biol. Chem. 265 (1990) 9033-9042.

[95] J.I. MacRae, S. Lopaticki, A.G. Maier, T. Rupasinghe, A. Nahid, A.F. Cowman, et al., P lasmodium falciparum is dependent on de novo myo -inositol biosynthesis for assembly of GPI glycolipids and infectivity, Mol. Microbiol. 91 (2014) 762-776. doi:10.1111/mmi.12496.

[96] A. Conzelmann, H. Riezman, C. Desponds, C. Bron, A major 125-kd membrane glycoprotein of Saccharomyces cerevisiae is attached to the lipid bilayer through an 
inositol-containing phospholipid., EMBO J. 7 (1988) 2233-40.

[97] P. Orlean, Dolichol phosphate mannose synthase is required in vivo for glycosyl phosphatidylinositol membrane anchoring, 0 mannosylation, and $\mathrm{N}$ glycosylation of protein in Saccharomyces cerevisiae., Mol. Cell. Biol. 10 (1990) 5796-805. doi:10.1128/MCB.10.11.5796.

[98] E. Di Daniel, M.H. Mok, E. Mead, C. Mutinelli, E. Zambello, L.L. Caberlotto, et al., Evaluation of expression and function of the $\mathrm{H}+$ /myo-inositol transporter HMIT, BMC Cell Biol. 10 (2009) 54. doi:10.1186/1471-2121-10-54.

[99] R.H. Michell, Inositol derivatives: evolution and functions., Nat. Rev. Mol. Cell Biol. 9 (2008) 151-61. doi:10.1038/nrm2334.

[100] J.C. Wright, D.W. Hood, G.A. Randle, K. Makepeace, A.D. Cox, J. Li, et al., lpt6, a gene required for addition of phosphoethanolamine to inner-core lipopolysaccharide of Neisseria meningitidis and Haemophilus influenzae, J Bacteriol. 186 (2004) 69706982. doi:186/20/6970 [pii]10.1128/JB.186.20.6970-6982.2004.

[101] H.-G. Joost, G.I. Bell, J.D. Best, M.J. Birnbaum, M.J. Charron, Y.T. Chen, et al., Nomenclature of the GLUT/SLC2A family of sugar/polyol transport facilitators., Am. J. Physiol. Endocrinol. Metab. 282 (2002) E974-6. doi:10.1152/ajpendo.00407.2001.

[102] S. Schneider, Inositol transport proteins., FEBS Lett. 589 (2015) 1049-58. doi:10.1016/j.febslet.2015.03.012.

[103] A. Leber, C. Hrastnik, G. Daum, Phospholipid-synthesizing enzymes in Golgi membranes of the yeast, Saccharomyces cerevisiae., FEBS Lett. 377 (1995) 271-4.

[104] C.R. McMaster, R.M. Bell, CDP-ethanolamine:1,2-diacylglycerol ethanolaminephosphotransferase., Biochim. Biophys. Acta. 1348 (1997) 117-23.

[105] A. Mancini, F. Del Rosso, R. Roberti, P. Orvietani, L. Coletti, L. Binaglia, Purification of ethanolaminephosphotransferase from bovine liver microsomes., Biochim. Biophys. Acta. 1437 (1999) 80-92.

[106] A. Hartmann, M. Hellmund, R. Lucius, D.R. Voelker, N. Gupta, Phosphatidylethanolamine Synthesis in the Parasite Mitochondrion Is Required for Efficient Growth but Dispensable for Survival of Toxoplasma gondii., J. Biol. Chem. 289 (2014) 6809-24. doi:10.1074/jbc.M113.509406.

[107] S. Déchamps, K. Wengelnik, L. Berry-Sterkers, R. Cerdan, H.J. Vial, L. Gannoun-Zaki, 
Lipid topogenesis - 35 years on

The Kennedy phospholipid biosynthesis pathways are refractory to genetic disruption in Plasmodium berghei and therefore appear essential in blood stages, Mol. Biochem. Parasitol. 173 (2010) 69-80. doi:10.1016/j.molbiopara.2010.05.006.

[108] L. Farine, M. Niemann, A. Schneider, P. Bütikofer, Phosphatidylethanolamine and phosphatidylcholine biosynthesis by the Kennedy pathway occurs at different sites in Trypanosoma brucei., Sci. Rep. 5 (2015) 16787. doi:10.1038/srep16787. 


\section{Graphical Abstract}

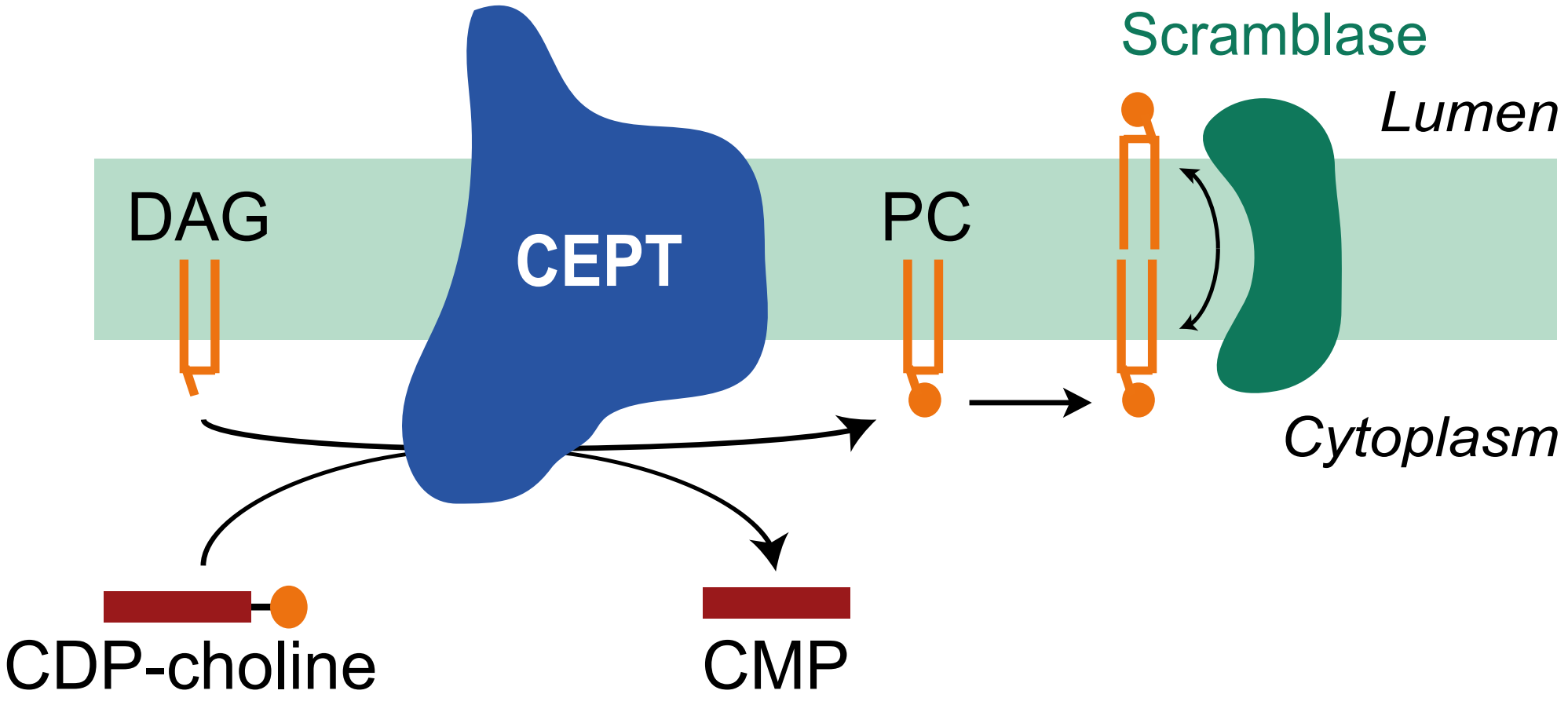

